\title{
5.04
}

\section{Mudanças na Gestão da Assistência Farmacêutica dos Componentes Especializados no Estado da Paraíba a partir da Implantação do HORUS.}

Changes in the Pharmaceutical Care Management Components Specialized in Paraiba State from the Implementation of HORUS.

\section{Fagner Dayan de Lima Gomes}

Estudante, Graduação (em curso), Universidade Federal da Paraíba. João Pessoa, Brasil.

\section{Marjorie Diony Abrantes Fernandes dos Santos}

Estudante, Graduação (em curso), Universidade Federal da Paraíba. João Pessoa, Brasil.

\section{Rossana Maria Souto Maior Serrano}

Professora Adjunta, Doutorado em Educação, Universidade Federal da Paraíba. João Pessoa, Brasil.

Resumo: Este estudo é um trabalho de pesquisa que compõe o projeto SIMSUSSistema de Informação sobre Medicamentos do SUS: acessibilidade e uso racional, e analisa o processo de organização do trabalho da Assistência Farmacêutica, e os aspectos referentes à acessibilidade aos medicamentos dos componentes especializados da Assistência Farmacêutica. É um estudo descritivo e analítico em que se descrevem as mudanças ocorridas na gestão quando da implantação do Sistema Nacional de Gestão da Assistência Farmacêutica - Hórus e analisa a pertinência das mudanças ocorridas. Como uma ferramenta basal para superar alguns dos maiores desafios para a assistência à saúde da população em todo o país, o Hórus, sistema criado pelo Ministério da Saúde em 3 de novembro de 2009, disponibiliza para todos os níveis de governo, um software que permite a integração de informações entre as farmácias, unidades de saúde e almoxarifado. O Centro Especializado de Dispensação de Medicamentos Excepcionais (CEDMEX) do Estado da Paraíba implantou o sistema Hórus em 20 de julho de 2012, e em razão dessa implantação modificou seus processos de trabalho para atender aos 23.160 pacientes cadastrados. O Centro fornece 149 medicamentos com 321 apresentações para 
atender a 79 patologias. O estudo busca investigar a resposta dada pela implantação do HÓRUS, os benefícios ocorridos após sua implantação, as mudanças ocorridas na gestão, os aspectos referentes aos processos de requisição e de organização do serviço. O estudo foi desenvolvido a partir da análise da literatura sobre o sistema e entrevista com os gestores envolvidos em sua implantação, bem como a análise dos documentos normativos oficiais. Mesmo considerando que o processo ainda não está concluso registram-se mudanças significativas no campo do planejamento, controle e monitoramento e da acessibilidade.

Palavras chave: Assistência farmacêutica; Gestão em Saúde; acessibilidade ao medicamento.

Keywords: Pharmaceutical Services; Health Management; accessibility to medicines

\section{Introdução}

O custo saúde no Brasil tem sido historicamente discutido nos aspectos de eficiência dos programas de prevenção e tratamento. Dentro do conceito de eficiência têm sido discutidos os mecanismos de monitoramento e controle desses programas, objetivando a racionalidade dos processos de trabalho, a razoabilidade no uso dos recursos de saúde e a resolubilidade das ações.

O processo de gestão do trabalho em saúde exige, nos tempos atuais, que os programas e ações sigam padrões de base técnico/cientifica, sejam auditáveis, e primem pela celeridade nas respostas aos problemas de saúde. Assim sendo, a rede SUS capitaneada pelo Ministério da Saúde tem nos últimos anos buscado aperfeiçoar os processos de trabalho e gestão, e vem fomentando a implementação de mecanismos de monitoramento e controle dos processos em saúde.

A área de informação em saúde no Brasil, historicamente, foi condicionada por ações técnicas e tecnológicas, de forma centralizada, verticalizada e com baixo grau de interlocução e automação. Esse processo resultou em uma política de informação em saúde de caráter prescritivo, normativa, não participativa, segmentada segundo a lógica de programas específicos e pouco eficiente. Registrando-se a pulverização e duplicação dos sistemas de informação em saúde e ausência de comunicação entre instâncias central, regionais e locais, restringindo assim o papel dos estados, municípios e representações da sociedade na tomada de decisões (Mello Jorge, 2010).

Na medida em que os processos realizados no SUS têm ampla abrangência, envolvem saberes diversos e ações em vários campos, é imprescindível o uso da 
tecnologia para garantir o controle dos processos não só do ponto de vista de gestão, mas também por meio de orientações técnicas e estruturação da rede.

Com o desenvolvimento tecnológico no campo da informação e com a necessidade política e social de se estabelecer mecanismos de controle no campo da assistência farmacêutica, entre as diversas medidas de monitoramento dos processos de trabalho e uso de insumos pelo SUS destacamos o desenvolvimento no ano de 2009 do Sistema Nacional de Gestão da Assistência Farmacêutica - Hórus, que vem sendo gradualmente implementado nos estados da federação. A necessidade de um sistema computadorizado e que cubra o vasto aparelho de distribuição e dispensação de medicamentos é obviamente necessário em razão da grande demanda existente no SUS.

A implementação do Hórus tem gerado respostas importantes no campo da gestão, na medida em que tem possibilitado o melhor uso da informação para tomada de decisões e pactuação entre união, estados e municípios na cobertura do arsenal terapêutico necessário à saúde.

A implentação do Hórus pelo governo do Estado da Paraíba para gestão da assistência farmacêutica iniciou-se em 2012 e ainda encontra-se em processo de aperfeiçoamento, constituindo-se uma realidade instituída, que tem demandado mudanças e adequações nos processos de trabalho do Núcleo de Assistência Farmacêutica do Estado e no Centro Especializado de Dispensação de Medicamentos Excepcionais (CEDMEX).

Este trabalho busca identificar a resposta dada pela implantação do HÓRUS, os benefícios ocorridos após sua implantação, as mudanças ocorridas na gestão, os aspectos referentes aos processos de requisição e de organização do serviço.

\section{Metodologia}

É um estudo exploratório e descritivo, onde foi realizada uma revisão na literatura buscando identificar os fundamentos políticos e legais que pautaram a implantação do sistema Hórus, e a realização de entrevista aberta com gestores estaduais, principalmente do Centro Especializado de Dispensação de Medicamentos Excepcionais (CEDMEX) do Estado da Paraíba, onde buscou identificar as mudanças ocorridas na rede estadual a partir dessa realidade política e legal. 


\section{Resultados}

\section{As bases políticas para implantação do Hórus}

Garantir o acesso ao medicamento de forma segura tem sido um dos grandes problemas enfrentados pelo Sistema Único de Saúde, na medida em que saúde é um direito de todos e um dever do Estado e que a assistência farmacêutica é parte desse direito básico. Constitui-se um grande desafio a construção de um modelo de assistência farmacêutica que rompa preconcepções historicamente reproduzidas, e que demande um novo paradigma que promova benefícios sociais no campo técnico e econômico, pelo uso racional de medicamentos e pela racionalização da gestão, principalmente pública.

O Brasil vem construindo um modelo de Assistência Farmacêutica de forma gradual, porém expressiva, a partir da criação da Agencia Nacional de Vigilância Sanitária (ANVISA) (1999); da elaboração da Política Nacional de Medicamentos (PNM) (2001); com a criação do Departamento de Assistência Farmacêutica (DAF), e da Secretaria de Ciência e Tecnologia e Insumos Estratégico (SCTIE), em 2003; da aprovação da Política Nacional de Assistência Farmacêutica (PNAF) (2004); e implantação do Programa de Componentes Especializados da Assistência Farmacêutica, em 2009; tais marcos estruturais e políticos vêm gerando uma base normativa que fortalece a necessidade de melhorias e aperfeiçoamento no processo de gestão dos medicamentos, que buscaram resolver os problemas relacionados ao acesso e uso racional de medicamentos.

Não raro são os relatos e denuncias públicas de desabastecimentos dos serviços farmacêuticos; de perdas de medicamentos pelo vencimento da validade; de consumo excessivo, isso tem como principal causa as dificuldades de gestão dos processos farmacêuticos. Constata-se, nas rotinas dos serviços de saúde, que na maioria das vezes, as decisões políticas suplantam as técnicas e, além disso, os procedimentos operacionais são burocráticos e conservadores; as informações técnicas existentes são pouco consideradas no momento de planejamento, de modo que o pensamento sobre a resolubilidade no campo da assistência farmacêutica fica num nível de acomodação em face das dificuldades conjunturais e estruturais da rede.

Problemas estruturais como centralização do poder decisório, infraestrutura precária, mecanismos de controle burocráticos podem ser superados na medida em 
que haja decisão política para tal, e em que seja implantado um modelo de gestão horizontalizado em seu processo decisório, fundamentado em informações técnicas, e monitorado por um sistema de controle que gere informações confiáveis para a tomada de decisão.

Assim, a qualidade dos indicadores de saúde impõe-se como condição para a gestão das políticas públicas e para a tomada de decisões. Para assegurar tal qualidade, cumpre considerar alguns requisitos. A introdução de inovações em tecnologias de saúde deve estar associada às tecnologias de informação em saúde. Porém, deve também traduzir-se em indicadores de saúde e possibilitar a integração com sistemas nacionais e internacionais de indicadores de saúde e assim favorecer a comparação com diferentes séries históricas (Mello Jorge, 2010).

O uso de indicadores de assistência à saúde e de assistência farmacêutica em diferentes países vem se ampliando desde as últimas décadas do século $X X$, visando aferir o desempenho de sistemas de serviços de saúde. No Brasil, esse crescimento vem sendo impulsionado pela dinâmica de expansão dos sistemas de serviços de saúde, pelas demandas de seus usuários, no setor governamental e privado, e pela introdução de políticas públicas de saúde (Schout, 2007).

A conciliação entre fornecimento e demanda deve constituir-se numa meta a ser atingida. Essa conciliação deve dar respostas aos seguintes questionamentos: Qual a necessidade real? Como definir os produtos farmacêuticos que devem compor a padronização de medicamentos da rede? O que comprar? Quando comprar? Que critérios utilizar para seu uso? Como monitorar? Como avaliar as respostas geradas? são questões básicas para uma boa gestão da assistência farmacêutica, que deve ser pautada por informações técnico-cientifica de base farmacoepidemiológica.

Nesse sentido, inserida no complexo sistema de produção da saúde, a análise da política de assistência farmacêutica não se restringe aos processos organizacionais da aquisição, armazenagem ou distribuição de medicamentos, envolvendo uma avaliação mais ampliada da qual esses elementos fazem parte.

O principal desafio na busca da melhoria da assistência farmacêutica é possibilitar aos gestores da Política de Medicamentos o controle do processo em todas as instâncias decisórias, respeitando as competências de cada setor, mas estabelecendo mecanismos de avaliação de desempenho das unidades. Assim, um 
sistema de dados que possibilite a construção de indicadores claros e objetivos tem uma importância fundamental como uma ferramenta de trabalho.

Para suprir a lacuna de uma base de dados para a assistência farmacêutica, o governo federal adquire, em 2009, os direitos sobre o Sistema de Controle de Medicamentos. No Portal da Saúde (2013), o Hórus é definido como um sistema "fruto da parceria estabelecida, em 2009, entre o Departamento de Assistência Farmacêutica do Ministério da Saúde (DAF/MS); a Secretaria Municipal de Saúde de Recife (SMS/PE); a Empresa Pública de Informática do Recife (Emprel); o Departamento de Informática do SUS (DataSUS/MS); o Conselho Nacional de Secretários de Saúde (CONASS) e o Conselho Nacional de Secretarias Municipais de Saúde (CONASEMS)".

Entre os objetivos, o portal saúde destaca a qualificação da gestão da assistência farmacêutica, e aponta que as três esferas de governo que poderão:

“• conhecer o perfil de acesso e utilização dos medicamentos pelos usuários dos serviços de saúde;

- contribuir para a formação de um sistema nacional de informações em assistência Farmacêutica no SUS;

- contribuir para qualificar a atenção à saúde da população assistida pelo SUS;

- contribuir para a ampliação do acesso e da promoção do uso racional de medicamentos;

- aperfeiçoar os mecanismos de controle e aplicação dos recursos financeiros." (Portal Saúde, 2013).

É preciso contextualizar que a implantação do Hórus se dá dentro de um sistema organizado em linhas de cuidado e em componentes da assistência farmacêutica para oferecer medicamentos à população, quais sejam: Componente Básico, que dispõe de uma lista de mais de 450 medicamentos para atender a 90\% das demandas da atenção básica de saúde; Componente Especializado da assistência farmacêutica; e Componente Estratégico.

Dentro de cada um desses componentes, cada instância de governo tem competências definidas em lei e participa do processo de financiamento.

Assim, ao descrever o Hórus, o Portal Saúde (2013) aponta para as necessidades de gestão da cada componente, quais sejam: 


\section{- Componente Básico da Assistência Farmacêutica: HÓRUS Básico}

Possibilita o registro do fluxo de medicamentos e insumos em toda a rede de saúde municipal, bem como entre estados e municípios, incluindo as regionais estaduais de saúde. Tem como objetivo qualificar a gestão e execução do Componente Básico da Assistência Farmacêutica, por meio de cadastro único dos usuários, bem como dos medicamentos e insumos. O HÓRUS Básico também disponibiliza uma série de temas selecionados, reunidos sob o título de "Condutas Baseadas em Evidências sobre Medicamentos Utilizados em Atenção Primária à Saúde”, além de outras publicações elaboradas no intuito de disponibilizar às equipes de saúde informações para fundamentar a seleção, prescrição e dispensação de medicamentos essenciais, contribuindo para o uso racional de medicamentos.

- Componente Especializado da Assistência Farmacêutica: HóRUS

\section{Especializado}

Possibilita o registro do fluxo de medicamentos em toda a rede estadual que gerencia o Componente Especializado da Assistência Farmacêutica; cadastro único de pacientes com possibilidade de consulta aos usuários cadastrados no HÓRUS Básico; geração eletrônica dos arquivos da APAC; cálculo das doses de ataque para os medicamentos com regime posológico diferenciado; parametrização de todos os critérios dos Protocolos Clínicos e Diretrizes Terapêuticas, facilitando a avaliação das solicitações, entre outros. Tem como objetivo qualificar a gestão e execução do Componente Especializado da Assistência Farmacêutica. Substituirá gradativamente o Sismedex, atual sistema do Ministério da Saúde utilizado para a gestão do Componente Especializado da Assistência Farmacêutica uma vez que permite reunir informações acerca do consumo dos medicamentos fornecidos pelo SUS, constantes nos elencos do Componente Especializado da Assistência Farmacêutica.

- Componente Estratégico da Assistência Farmacêutica: HÓRUS

\section{Estratégico}

Permite executar e/ou acompanhar ações de gestão da Assistência Farmacêutica dos medicamentos do Componente Estratégico, cujo financiamento e abastecimento são de responsabilidade da esfera federal. O projeto iniciou com os Programas Nacionais de Controle da Tuberculose e Hanseníase e encontra-se em fase de levantamento dos requisitos e definição das regras de negócio para posteriormente atender os demais Programas de Saúde do componente (DST/AIDS, 
malária, leishmaniose, doença de chagas, esquistossomose, cólera, outras doenças endêmicas de abrangência nacional ou regional; alimentação e nutrição, tabagismo, sangue e hemoderivados; e imunobiológicos).

O HÓRUS está integrado ao Cartão Nacional de Saúde e ao Cadastro Nacional de Estabelecimentos de Saúde e, apesar de sua adesão não ser obrigatória por parte dos estados e municípios brasileiros, está apto a integrar os diferentes sistemas de controle e gestão de medicamentos no âmbito do Ministério da Saúde e interoperar com os sistemas estaduais e municipais, visando à construção de um banco nacional de informação da assistência farmacêutica no SUS.

Apesar de ser um sistema de abrangência nacional ainda em implantação, Costa e Nascimento Jr (2012), identificaram em seus estudos mudanças no processo de gestão da assistência farmacêutica, quais sejam:

Quadro 1. Síntese das mudanças após a implantação do Hórus nos municípios. Brasil, dezembro de 2009 a dezembro de 2011.

\begin{tabular}{lll}
\hline Categoria & Situação anterior & Situação após implantação do Hórus \\
\hline Controle de estoque & $\begin{array}{l}\text { Ausência ou deficiência no controle do } \\
\text { estoque }\end{array}$ & Controle em tempo real \\
& $\begin{array}{l}\text { Falta de controle no fluxo de } \\
\text { medicamentos }\end{array}$ & $\begin{array}{l}\text { Conhecimento do fluxo de medicamentos na rede } \\
\text { de saúde }\end{array}$ \\
& Falta de medicamentos & Redução da descontinuidade \\
Programação & Programação inadequada & $\begin{array}{l}\text { Programação baseada em consumo real/demanda } \\
\text { atendida e programada }\end{array}$ \\
Controle do prazo devalidade & $\begin{array}{l}\text { Medicamentos com data de validade } \\
\text { vencida ou prestes a vencer }\end{array}$ & $\begin{array}{l}\text { Diminuição das perdas e possibilidade de } \\
\text { remanejamentos }\end{array}$ \\
Seleção de medicamentos & $\begin{array}{l}\text { Desconhecimento do perfil de consumo } \\
\text { da população }\end{array}$ & $\begin{array}{l}\text { Identificação do perfil de consumo de } \\
\text { medicamentos no território }\end{array}$ \\
& $\begin{array}{l}\text { Desconhecimento do perfil de } \\
\text { prescrição }\end{array}$ & $\begin{array}{l}\text { Identificação do perfil de prescrição de } \\
\text { medicamentos no território }\end{array}$ \\
Recursos humanos & $\begin{array}{l}\text { Lacunas na atualização das normas/ } \\
\text { procedimentos da gestão da Assistência } \\
\text { Farmacêutica }\end{array}$ & $\begin{array}{l}\text { Atualização das normas, procedimentos, } \\
\text { diretrizes para a organização dos serviços de } \\
\text { Assistência Farmacêutica }\end{array}$ \\
Inexistência ou insuficiência de & $\begin{array}{l}\text { Informações fidedignas em tempo real para } \\
\text { subsidiar a tomada de decisão }\end{array}$ \\
\hline
\end{tabular}

Fonte: Costa e Nascimento Jr., 2012

Tais mudanças reforçam o papel estratégico do Hórus como ferramenta de gestão e por consequência uma visão mais ampliada sobre a gestão da assistência farmacêutica, na medida em que este aponta as fragilidades e necessidades da rede. 


\title{
O contexto do Hórus na gestão estadual da Paraíba
}

A situação do Estado da Paraíba no tocante a reorientação da Assistência Farmacêutica não difere da maioria dos Estados e ainda está em processo de construção, entretanto a realidade do Estado tem se modificado a partir das lutas e embates internos nesse campo, e as mudanças de paradigmas ocorridas na Política Nacional de Medicamentos.

$\mathrm{Na}$ análise da estrutura de gestão do Estado encontramos que até o ano de 2004 não havia na estrutura formal da Secretaria Estadual de Saúde uma coordenação que consolidasse as propostas e definisse as diretrizes da Política Estadual de Medicamentos e Assistência Farmacêutica. A rede estava organizada de forma fragmentada em setores que respondiam por segmentos da assistência farmacêutica, o que impossibilitava a gestão sistêmica dos medicamentos.

\begin{abstract}
Essa ausência gerou uma indefinição na formulação da Política Estadual de Medicamentos levando a uma fragmentação das ações de Assistência Farmacêutica em diversos programas cada um com uma lógica e uma logística diferente, causando sobreposição dos serviços, indefinição das responsabilidades, e contribuindo para um desempenho inexpressivo que em última instância prejudicava 0 acesso ao medicamento. (Medeiros, 2012)
\end{abstract}

A Secretaria de Saúde do Estado da Paraíba - SES tem como missão definir, coordenar e implantar a política estadual de saúde em consonância com os princípios e diretrizes do SUS com vistas à promoção da saúde da população. Como gestora da política estadual de assistência farmacêutica a SES-PB pode tornar sua atuação mais decisiva envolvendo todos os municípios e assim consolidar definitivamente a implementação das diretrizes da Política Nacional de Medicamentos, entre elas a reorientação da Assistência Farmacêutica. (Medeiros, 2012)

A Secretaria Estadual de Saúde promove mudanças em sua estrutura de gestão criando em 2011 o Núcleo de Assistência Farmacêutica (NAF), instancia da Gerência Executiva de Atenção à Saúde (GEAS), e que tem um papel coordenador de todos os programas relacionados a medicamentos no Estado. Cabe ao NAF implantar as diretrizes da Política Nacional de Medicamentos e Assistência Farmacêutica em parceria com os municípios paraibanos.

$\mathrm{Na}$ pesquisa realizada para esse estudo observou-se e foi relatado que 0 processo de controle de medicamentos na gestão da assistência farmacêutica estadual se dava de forma informatizada, através do Sistema de Medicamentos de 
Dispensação Excepcional (SISMEDEX), portanto apenas para um dos componentes da assistência farmacêutica. Com a implantação do Hórus, a cobertura se amplia e possibilita o monitoramento do consumo dos medicamentos com maior agilidade.

A partir do maior controle estabelecido pelo Hórus ficou evidenciada a necessidade de novas normatizações e o estabelecimento e uso de protocolos clínicos para a análise das demandas por medicamentos, como exemplo destaca-se a Portaria no 1554 , de 30 de julho de 2013, que dispõe sobre os componentes especializados.

Alem da nova portaria o Hórus também trouxe para o centro e sua administração mudanças significativas, que de maneira geral o antigo sistema não supria, pois por conta de suas limitações abrangia apenas a farmácia básica; muitas das mudanças alcançadas foram:

- $\quad$ a concepção na preparação de referentes ${ }^{1}$ de Assistência Farmacêutica;

- a autorização por intervenções em tempo real- gerenciamento estratégico em qualquer ponto do município;

- $\quad$ o aperfeiçoamento da gestão da Assistência Farmacêutica;

- $\quad$ permitiu a rastreabilidade dos medicamentos;

- $\quad$ permitiu a concretização de estudos de utilização de medicamentos;

- $\quad$ proporcionou maior disponibilidade do profissional farmacêutico para as atividades assistenciais e de treinamento.

Observa-se que, para a implantação do Hórus e a partir do seu estabelecimento na rede estadual, ocorreram mudanças, em maior ou menor dimensão, nos seguintes campos:

- $\quad$ formulação de políticas;

- $\quad$ estabelecimento de prioridades;

- $\quad$ construção e implementação de um matriz de alocação de recursos;

- $\quad$ modernização e flexibilização da estrutura organizacional

\footnotetext{
1 Nota: No quadro de pesquisa, são instrumentos de gestão essenciais nas atividades de monitoramento e avaliação. Aqui se refere aos indicadores de qualidade como uma ferramenta na gestão de dispensasão de medicamentos, maximizando a assistência farmacêutica e contribuindo para a melhoria contínua de processos organizacionais.
} 
O principal desafio na busca da melhoria do sistema é possibilitar aos gestores da Política de Medicamentos a participação nos processos em todas as instâncias decisórias, respeitando as competências de cada setor, mas estabelecendo mecanismos de avaliação de desempenho das unidades.

Sabe-se que a qualidade dos serviços se mede em razão da qualidade da estrutura, dos processos e dos resultados nessa perspectiva, apontamos para a necessidade de desenvolvimento de uma estratégia e modelo de organização do trabalho que contemplem os seguintes aspectos:

- melhoramento no processo logístico, buscando, a partir da necessidade sanitária real, estabelecer procedimentos de aquisição, armazenagem e distribuição racionais e eficazes;

- implantação de uma estrutura organizacional flexível, menos burocrática, com estrutura lógica de articulações entre as unidades e que possibilite respostas ágeis e oportunas;

- fomento a implantação do Hórus em todos os municípios do Estado, com a incorporação dos indicadores construídos no processo de planejamento e que possa construir uma rede que dialogue sobre esses indicadores.

Para viabilizar essas alterações, são necessárias as seguintes medidas: elaboração e implementação de um programa de capacitação de recursos humanos; implantação efetiva do Hórus, que possibilite o controle dos processos e a obtenção de informações em tempo real; estabelecimento de rotinas escritas; controle técnico do processo de aquisição, armazenagem e distribuição de medicamentos; melhorias na infra-estrutura física e sua adequação às boas práticas de medicamentos.

\section{Considerações finais}

As mudanças de paradigma da assistência farmacêutica preconizadas pela Política Nacional de Assistência Farmacêutica é um processo em implantação na saúde pública brasileira, compreendemos que é necessária uma coerência entre o modelo preconizado e as respostas geradas pela gestão, sendo portanto necessário mudanças na forma de gestão e na estrutura e capacidade dos serviços serviço. Hoje não se pode pensar em gestão da assistência farmacêutica de forma improvisada e amadora, sendo necessário o aperfeiçoamento de processos e a implantação plena do Hórus como ferramenta de avaliação e controle. 
Com os resultados positivos que o Hórus tem alcançado é fácil imaginar que não demorará a instalação por completo em todas as regionais de saúde do Estado da Paraíba, porém é possível observar que é um sistema que ainda está em fase de aprimoramento, e que por mais importante que seja para o SUS precisa passar por ajustes através dos planejamentos e avaliações realizadas pelos gestores e atores que o operacionalizam.

\section{Referências}

BRASIL. Conselho Nacional de Saúde. Política nacional de assistência farmacêutica: CNS RES no 338/2004. Brasília: CDD (22ª ed.), 2004.

BRASIL. Constituição (1988). Constituição da República Federativa do Brasil. Brasília: Senado Federal, 1998.

BRASIL. Lei no 8.080, de 19 de setembro de 1990. Dispõe sobre as condições para a promoção, proteção e recuperação da saúde, a organização e o funcionamento dos serviços correspondentes e dá outras providências. Diário Oficial da União, Brasília, DF, 20 set. 1990. p. 18055.

BRASIL. Ministério da Saúde. Assistência farmacêutica: planejar, organizar, avaliar é preciso. Brasília: MS ed. 2006c.

BRASIL. Ministério da Saúde. Política nacional de medicamentos: Portaria GM/MS n.ำ 3.916,/98. Brasília: Diário Oficial (da) República Federativa do Brasil, 1998.

COSTA, K. S. e NASCIMENTO Jr, J. M. HÓRUS: Inovação tecnológica na Assistência Farmacêutica no Sistema Único de Saúde. Revista de Saúde Pública, 9 págs., 2012. Disponível em http://www.scielosp.org/pdf/rsp/2012nahead/ao4223.pdf , acessado em agosto de 2013.

MEDEIROS, K. M. A Reorientação da Assistência Farmacêutica no Estado da Paraíba como diretriz da Política Estadual de Medicamentos: o caso da judicialização do acesso a medicamentos através da Secretaria Estadual de Saúde. Trabalho monográfico, João Pessoa, 2012.

MELLO JORGE MHP, LAURENTI R, GOTLIEB SLD. Avaliação dos sistemas de informação em saúde no Brasil. Cad Saude Coletiva; 18(1):7-18, 2010.

MINISTÉRIO DA SAÚDE, Portal da Saúde. Disponível em http://portalsaude.saude.gov.br/portalsaude/index.html, acessado em 10 de agosto de 2013

SCHOUT D, NOVAES HMD. Do registro ao indicador: gestão da produção da informação assistencial nos hospitais. Cienc Saude Coletiva; 12(4):935-44, 2007. DOI: 10.1590/S1413-81232007000400015 
Anais dos III Congresso Iberoamericano de Direito Sanitário / II Congresso Brasileiro de Direito Sanitário

WATERS, E. et al. Evaluating the effectiveness of public health interventions: the role and activities of the Cochrane Collaboration. J. Epidemiol. Community Health, London, 60(4):285-289, 2006. 